\title{
Vacancy clustering and prismatic dislocation loop formation in aluminum
}

\author{
Vikram Gavini \\ Department of Mechanical Engineering, University of Michigan, Ann Arbor, Michigan 48109, USA
}

Kaushik Bhattacharya and Michael Ortiz

Division of Engineering and Applied Science, California Institute of Technology, Pasadena, California 91125, USA

(Received 9 October 2007; published 15 November 2007)

\begin{abstract}
The formation of prismatic dislocation loops is an important factor leading to radiation damage of metals. However, the formation mechanism and the size of the smallest stable loop has remained unclear. In this Rapid Communication, we use electronic structure calculations with millions of atoms to address this problem in aluminum. Our results show that there is a cascade of larger and larger vacancy clusters with smaller and smaller energy. Further, the results show that a seven vacancy cluster on the (111) plane can collapse into a stable prismatic loop. This supports the long-standing hypothesis that vacancy clustering leads to a prismatic loop, and that these loops can be stable at extremely small sizes. Finally our results show that it is important to conduct calculations using realistic concentrations (computational cell size) to obtain physically meaningful results.
\end{abstract}

DOI: 10.1103/PhysRevB.76.180101

PACS number(s): 61.82.-d, 02.70.-c, 31.15.Ew, 61.72.Lk

The embrittlement of metals subjected to radiation is a long-standing problem in various applications including nuclear reactors. As the irradiation dose increases above a certain threshold, a significant population of prismatic dislocation loops (dislocation loops whose Burgers vector has a component normal to their plane) has been experimentally observed to arise in metals. ${ }^{1-5}$ It is widely believed these prismatic loops form through the clustering of vacancies that are generated randomly by irradiation. ${ }^{6}$ Specifically, the vacancies diffuse and eventually cluster on specific planes. Once there is a large enough planar cluster, the atoms on the two faces collapse onto each other leaving behind a prismatic dislocation loop.

However, the formation mechanism and the size of the smallest stable loop remain unclear: there is no direct experimental observation of the process, and the theoretical investigations are inconclusive. Recent molecular dynamics simulations $^{7}$ support the hypothesized mechanism for iron, but these calculations used the Finnis-Sinclair empirical atomistic potentials whose validity is uncertain in situations involving changing atomic bonds. ${ }^{8}$ In contrast, calculations for aluminum using quantum mechanical density-functional theory 9,10 show that divacancies - a complex of two vacancies - are either energetically unfavorable if they are aligned along the $\langle 110\rangle$ direction or barely favorable with negligible binding energy if aligned along $\langle 100\rangle$. If two vacancies can barely bind, it seems doubtful that they can be stable and grow to form clusters that can turn into prismatic loops. While these density-functional theory (DFT) methods are far more accurate, the computational effort is extremely large and consequently these calculations were limited to less than 100 atoms. This corresponds to an unphysically high concentration of vacancies. Furthermore, the results are in variance with experiments ${ }^{11,12}$ that are indicative of a high binding energy of divacancies and a significant concentration of divacancies, especially at elevated temperatures.

We study vacancy clustering and prismatic loops by performing electronic structure calculations using orbital-free density-functional theory (OFDFT). ${ }^{13}$ Specifically, the kinetic energy functional is modeled using the Thomas-FermiWeizsacker family of functionals with $\lambda=1 / 6$. We use the modified form of the Heine-Abarenkov pseudopotential ${ }^{14}$ for aluminum to model the external field created by the nuclei and core electrons. The exchange-correlation effects are treated using a local density approximation. ${ }^{15,16}$ These functionals have been shown to correctly predict the bulk and vacancy properties of aluminum. ${ }^{17,18}$

A challenge in studying defects in solids, and especially vacancies, is their extremely small concentrations which are typically a few parts per million in aluminum. ${ }^{19}$ Therefore any realistic calculation of vacancies and their interaction has to involve millions of atoms. Unfortunately, performing electronic structure calculations with such numbers of atoms remained beyond reach until the recent development of the quasicontinuum orbital-free density-functional method (QCOFDFT). ${ }^{18}$ This method has enabled the calculation of the electronic structure using OFDFT of samples with millions of atoms subjected to arbitrary boundary conditions. Importantly, the method is completely seamless, does not require any ad hoc assumptions, uses OFDFT as its only input, and enables convergence studies of its accuracy.

The independent unknowns of the QCOFDFT analysis are the nuclear positions and the electronic fields comprised of electron-density and electrostatic potential. As in the conventional QC approach, the nuclei positions are interpolated from the positions of representative nuclei, Fig. 1(a). Near the defect core, all nuclei are represented, whereas away from the defect core the interpolation becomes coarser and a small fraction of the nuclei determines the displacements of the rest. We refer to this computational mesh as the coarse mesh. The electronic fields exhibit subatomic oscillations and require a fine mesh to accurately capture these oscillations. In regions away from the defect cores these oscillations are locally periodic on the length scale of the lattice. Hence a first guess to the electronic fields is computed from a periodic unit cell calculation using the underlying CauchyBorn lattice deformation. We refer to this guess as the 


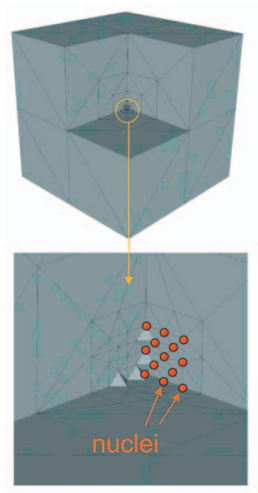

Coarse grid

(a)
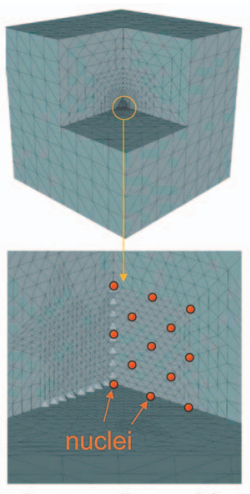

Intermediate grid

(b)

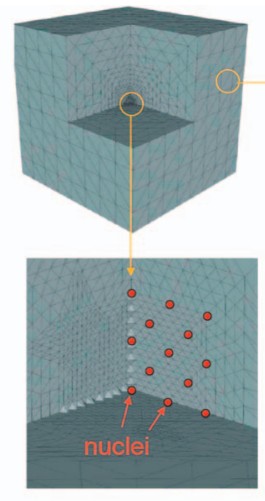

(c)

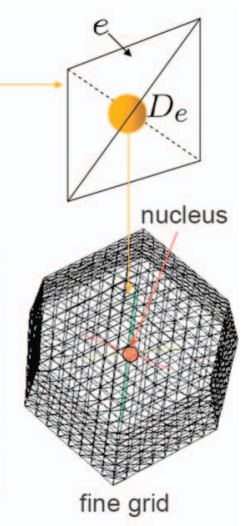

FIG. 1. (Color) (a) Coarse computational mesh used to interpolate nuclei positions away from the fully resolved defect core. (b) Intermediate computational mesh used to represent the corrector fields. It has subatomic resolution in the defect core, and coarsens away from the defect core. (c) Fine auxiliary mesh used to sample the Cauchy-Born predictor fields within an integration lattice unit cell, $D_{e}$, in each element $e$.
Cauchy-Born predictor. Though the Cauchy-Born predictor is an accurate representation of the electronic fields in regions far away from the defect, they greatly differ close to the defect cores. Thus the electronic fields are represented as a sum of the Cauchy-Born predictor and corrector fields. The corrector fields are computed on a mesh that exhibits subatomic resolution near the defect core, where the electronic fields differ greatly from the Cauchy-Born predictor, and coarsens away from the defect core, where the corrector fields exhibit slow variation on the scale of the lattice, Fig. 1(b). We refer to this computational mesh as the intermediate mesh. Finally, the Cauchy-Born predictor is sampled on an auxiliary mesh that resolves an integration lattice unit cell in each finite element with exceedingly fine resolution, Fig. 1(c). This auxiliary mesh is introduced for purposes of representing the Cauchy-Born predictor and does not introduce any degrees of freedom into the calculation. The degrees of freedom of the QCOFDFT analysis are the corrector fields on the intermediate mesh and the positions of representative atoms on the coarse mesh which are computed from a variational principle. These fields completely describe the electronic structure and the relaxed atomic structure of the material system.

In a recent work, we used QCOFDFT to study divacancies in aluminum, ${ }^{18}$ where we found a very strong cell-size (concentration) effect. Specifically, we found that $\langle 110\rangle$ di- vacancies were repulsive for small cell sizes, in agreement with previous calculations, ${ }^{9,10}$ and the same divacancies were attractive for larger cell sizes corresponding to realistic concentrations, with binding energies of $0.19 \mathrm{eV}$ in agreement with experimental measurements. ${ }^{11,12}$ This work showed that electronic structure calculations do not rule out vacancy clustering in aluminum. Therefore we examine this mechanism further in the current work.

We begin by examining the binding energies of various quadvacancies formed from a pair of divacancies. The number of possible quadvacancies that may be formed from a pair of divacancies is very large. Thus we restrict our analysis to configurations such that each vacancy has at least two other vacancies as nearest or second nearest neighbors. We shall justify this choice subsequently. This criterion results in nine distinct configurations (up to symmetry), six of which are planar vacancy clusters and three of which are nonplanar. These configurations are listed in Table I.

The vacancy cluster binding energy of an $n$-vacancy cluster is defined as

$$
E_{n v}^{b i n d}=n E_{v}^{f}-E_{n v}^{f}
$$

where $E_{v}^{f}$ denotes the formation energy of a single vacancy and $E_{n v}^{f}$ the formation energy of the $n$-vacancy cluster. The vacancy cluster binding energies for the nine configurations

TABLE I. Vacancy cluster binding energies for quadvacancies formed from a pair of divacancies. All possible quadvacancies such that each vacancy has two other vacancies as nearest or second nearest neighbors are considered.

\begin{tabular}{|c|c|c|c|}
\hline & Structure & Positions of vacancies & $\begin{array}{c}\text { Vacancy binding } \\
\text { energy }(\mathrm{eV})\end{array}$ \\
\hline 1 & planar $\{100\}$ & $(0,0,0),(a / 2, a / 2,0),(a, 0,0),(a / 2,-a / 2,0)$ & 0.52 \\
\hline 2 & planar $\{100\}$ & $(0,0,0),(a / 2, a / 2,0),(a, 0,0),(3 a / 2, a / 2,0)$ & 0.50 \\
\hline 3 & planar $\{100\}$ & $(0,0,0),(a / 2, a / 2,0),(a, 0,0),(a, a, 0)$ & 0.48 \\
\hline 4 & planar $\{100\}$ & $(0,0,0),(a, 0,0),(0, a, 0),(a, a, 0)$ & 0.48 \\
\hline 5 & planar $\{110\}$ & $(0,0,0),(0, a / 2, a / 2),(a, 0,0),(a, a / 2, a / 2)$ & 0.56 \\
\hline 6 & planar $\{111\}$ & $(0,0,0),(0, a / 2, a / 2),(a / 2, a / 2,0),(a / 2, a, a / 2)$ & 0.55 \\
\hline 7 & nonplanar & $(0,0,0),(0, a / 2, a / 2),(a / 2,0, a / 2),(a / 2, a / 2,0)$ & 0.53 \\
\hline 8 & nonplanar & $(0,0,0),(a, 0,0),(a / 2, a / 2,0),(a / 2,0, a / 2)$ & 0.51 \\
\hline 9 & nonplanar & $(0,0,0),(a, 0,0),(a / 2, a / 2,0),(0, a / 2, a / 2)$ & 0.50 \\
\hline
\end{tabular}




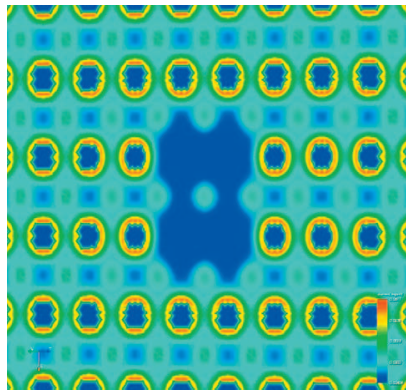

(a)

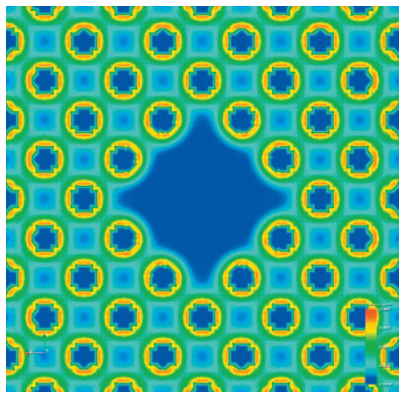

(b)
FIG. 2. (Color) (a) Contours of electron density around a planar quadvacancy (configuration No. 5 in Table I) on the (110) plane in a $10^{6}$ atom sample. This planar quadvacancy has the highest binding energy among the various quadvacancies considered. (b) Contours of electron density around a planar quadvacancy (configuration No. 1 in Table I) on a (100) plane in a $10^{6}$ atom sample.

of quadvacancies are tabulated in Table I. Figure 2(a) shows the contours of electron density for the quadvacancy cluster with the highest binding energy. This corresponds to configuration No. 5 in Table I, which denotes a planar quadvacancy on the (110) plane. Binding energies of each of these vacancy clusters listed in Table I are computed using a computational cell consisting of $10^{6}$ atoms. This corresponds to realistic vacancy concentrations of a few parts per million. ${ }^{19}$ The boundary conditions for all simulations are chosen such that the electronic fields decay to bulk values on the boundaries of the sample. Numerical parameters were chosen to keep the error in the formation energy due to discretization and coarse-graining to be less than $0.01 \mathrm{eV}$.

It is interesting to observe that all the quadvacancies considered have positive binding energies. Further, they also have binding energies larger than twice the computed divacancy binding energy of 0.19 and $0.23 \mathrm{eV}$ for $\langle 110\rangle$ and $\langle 100\rangle$ divacancies, respectively. This indicates that pairs of divacancies are attractive in all cases. These results suggest that quadvacancy formation is an energetically feasible process and that vacancies prefer to condense rather than split into mono- or divacancies. This observation also justifies our restriction to nine quadvacancy configurations.

The cell size used to simulate defects effectively sets the concentration of the defects. To understand the effect of vacancy concentration on the feasibility of vacancy clustering, we study the cell-size effect on quadvacancy binding energy for the first configuration in Table I. This configuration represents a square-shaped quadvacancy on the (100) plane, whose electronic structure is shown in Fig. 2(b). Figure 3 shows a strong dependence of the vacancy binding energy on the cell size. The quadvacancy which is energetically favorable for large cell sizes becomes unstable for small cell sizes. This is a result of the long-ranged elastic and electrostatic effects in the presence of these defects. This cell-size dependence shows that vacancy clustering which is feasible at low and realistic vacancy concentrations becomes unfavorable at high concentrations.

The results in Table I also show that the configurations with the highest binding energy (Nos. 5 and 6) are planar quadvacancy clusters on $\{110\}$ and $\{111\}$ planes. Therefore

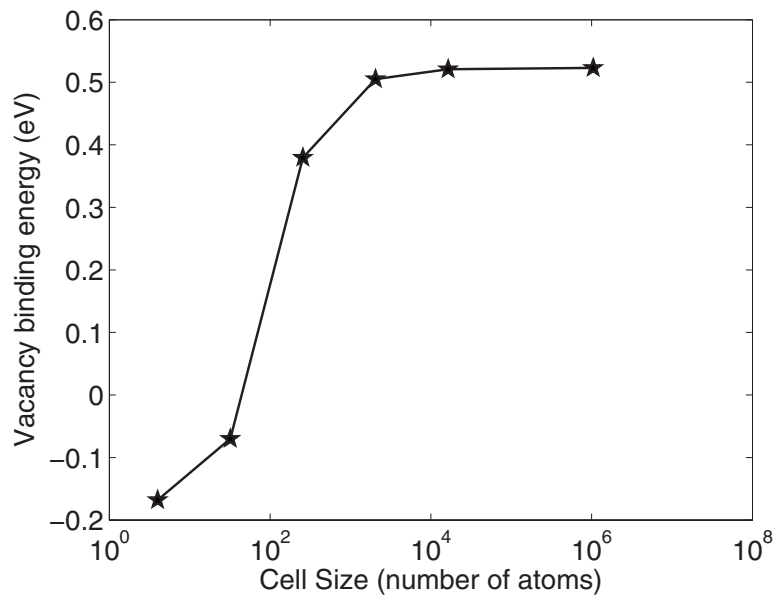

FIG. 3. Cell-size dependence of vacancy binding energy for the quadvacancy given by the first configuration in Table I.

we performed simulations on larger vacancy clusters on $\{110\}$ and $\{111\}$ planes, again using cell sizes with a $10^{6}$ atoms. On the (111) plane, we studied a hexagonal cluster with seven vacancies and found two stable configurations. One of the stable configurations is a noncollapsed state with a vacancy cluster binding energy of $0.88 \mathrm{eV}$, with a maximum displacement of atoms of the order of $3.2 \%$ of the nearest neighbor distance. Note that this is larger than $7 / 2$ times the divacancy binding energy ( 0.19 or $0.23 \mathrm{eV}$ depending on orientation). This means that the hexagonal cluster is stable against dissociation into divacancies.

The second configuration is a prismatic loop where the atoms above and below the hexagonal vacancy disk collapse or move toward each other leaving a dislocation line at the boundary of the disk. Figure 4 shows the atomic positions and the contours of the electron density on (001) and (111) planes of the collapsed prismatic loop. In particular, the dotted lines in Fig. 4(a) depict the collapse of the planes resulting in the prismatic dislocation loop. The maximum displacement of atoms is around $44 \%$ of the nearest neighbor distance, the Burgers vector is $0.44[110]$, and the dislocation plane is (111). These results are consistent with experiments. ${ }^{20,21}$ Using a transmission electron microscope (TEM), it was observed that prismatic loops form predominantly on a $\{111\}$ plane with a $1 / 2\langle 110\rangle$ Burgers vector. Fur-

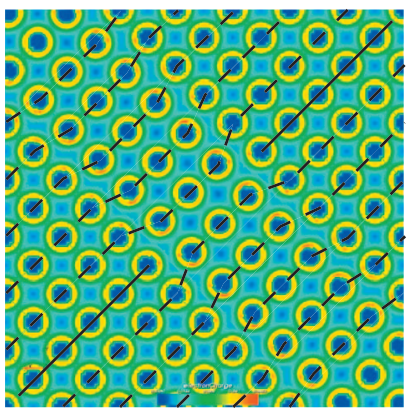

(a)

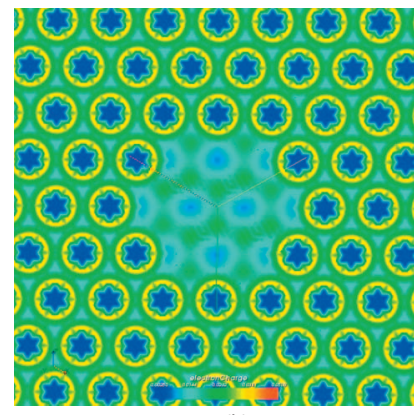

FIG. 4. (Color) (a) Contours of electron density on the (001) plane around a collapsed vacancy prismatic loop. (b) Contours of electron density around the prismatic loop on the (111) plane. 
ther, in these experiments, prismatic loops whose size is as small as $50 \AA$ in diameter were observed. While these are larger than our hexagonal prismatic loop formed from seven vacancies, it is impossible to detect a loop as it nucleates. Thus the nucleation size of a prismatic loop was hitherto unknown. The computed vacancy cluster binding energy for the prismatic loop is $1.55 \mathrm{eV}$ which means that not only is this structure stable against dissociation of divacancies, but that it is even more stable than the uncollapsed configuration.

On the (110) plane, we studied rectangular vacancy clusters with six and nine vacancies. The computed binding energies for these vacancy clusters are 0.81 and $1.16 \mathrm{eV}$, respectively. The maximum displacement of atoms in these vacancy clusters is around $4 \%$ of the nearest neighbor distance. These clusters did not display any bistability and collapse to prismatic loops.

These results point to four important facts. First, the binding energy of vacancy clusters on $\{110\}$ and $\{111\}$ planes in aluminum increases with the size of the vacancy cluster. Also, these vacancy clusters are all stable, i.e., vacancies prefer to condense rather than split into mono or divacancies. This is a numerical confirmation from an electronic structure perspective that vacancy clustering is energetically favorable. Second, we observe from direct numerical simulation that the hexagonal vacancy cluster on the (111) plane collapses to form a prismatic loop. This establishes from electronic structure calculations that vacancy clustering and collapse of the planes surrounding the vacancy cluster is a possible mechanism for the nucleation of prismatic dislocation loops. Third, our results point to the fact that vacancy clusters as small as seven vacancies can collapse to form stable prismatic loops on $\{111\}$ planes. Finally, our results show the importance of studying defects in solids at realistic concentrations.

The financial support of the Army Research Office under MURI Grant No. DAAD19-01-1-0517 and the support of the Department of Energy through Caltech's ASC Center for the Simulation of the Dynamic Response of Materials is gratefully acknowledged.
${ }^{1}$ B. C. Masters, Philos. Mag. 11, 881 (1965).

${ }^{2}$ B. L. Eyre and A. F. Bartlett, Philos. Mag. 12, 261 (1965); J. Nucl. Mater. 47, 143 (1973).

${ }^{3}$ T. J. Bullough, C. A. English, and B. L. Eyre, Proc. R. Soc. London, Ser. A 435, 85 (1991).

${ }^{4}$ H. Kawanishi and E. Kuramoto, J. Nucl. Mater. 149, 899 (1986).

${ }^{5}$ L. L. Horton and K. Farrell, J. Nucl. Mater. 122, 684 (1984).

${ }^{6}$ J. P. Hirth and J. Lothe, Theory of Dislocations (McGraw-Hill, New York, 1968).

${ }^{7}$ J. Marian, B. D. Wirth, and J. M. Perlado, Phys. Rev. Lett. 88, 255507 (2002).

${ }^{8}$ G. J. Ackland, D. J. Bacon, A. F. Calder, and T. Harry, Philos. Mag. A 75, 713 (1997).

${ }^{9}$ K. Carling, G. Wahnstrom, T. R. Mattsson, A. E. Mattsson, N. Sandberg, and G. Grimvall, Phys. Rev. Lett. 85, 3862 (2000).

${ }^{10}$ T. Uesugi, M. Kohyama, and K. Higashi, Phys. Rev. B 68, 184103 (2003).

${ }^{11}$ P. Ehrhart, P. Jung, H. Schultz, and H. Ullmaier, Atomic Defects in Metal, Landolt-Börnstein, New Series, Group 3, Vol. 25 (Springer-Verlag, Berlin, 1991).

${ }^{12}$ T. Hehenkamp, J. Phys. Chem. Solids 55, 907 (1994).

${ }^{13}$ R. G. Parr and W. Yang, Density-Functional Theory of Atoms and Molecules (Oxford University Press, New York, 1989).

${ }^{14}$ L. Goodwin, R. J. Needs, and V. Heine, J. Phys.: Condens. Matter 2, 351 (1990).

${ }^{15}$ D. M. Ceperley and B. J. Alder, Phys. Rev. Lett. 45, 566 (1980).

${ }^{16}$ J. P. Perdew and A. Zunger, Phys. Rev. B 23, 5048 (1981).

${ }^{17}$ V. Gavini, J. Knap, K. Bhattacharya, and M. Ortiz, J. Mech. Phys. Solids 55, 669 (2007).

${ }^{18}$ V. Gavini, K. Bhattacharya, and M. Ortiz, J. Mech. Phys. Solids 55, 697 (2007).

${ }^{19}$ M. J. Fluss et al., J. Phys. F: Met. Phys. 14, 2831 (1984).

${ }^{20}$ D. Kuhlmann-Wisdorf and H. G. F. Wilsdorf, J. Appl. Phys. 31, 516 (1960).

${ }^{21}$ J. Takamura and I. G. Greenfield, J. Appl. Phys. 33, 247 (1961). 